\title{
Simulation of Material Removal Efficiency with Ultrashort Laser Pulses
}

\author{
M. Feit, A. Komashko, A. Rubenchik, M. Perry, P. Banks
}

This paper was prepared for submittal to the

Conference on Laser Ablation '99

Goettingen, Germany

July 19-23, 1999

July 1999

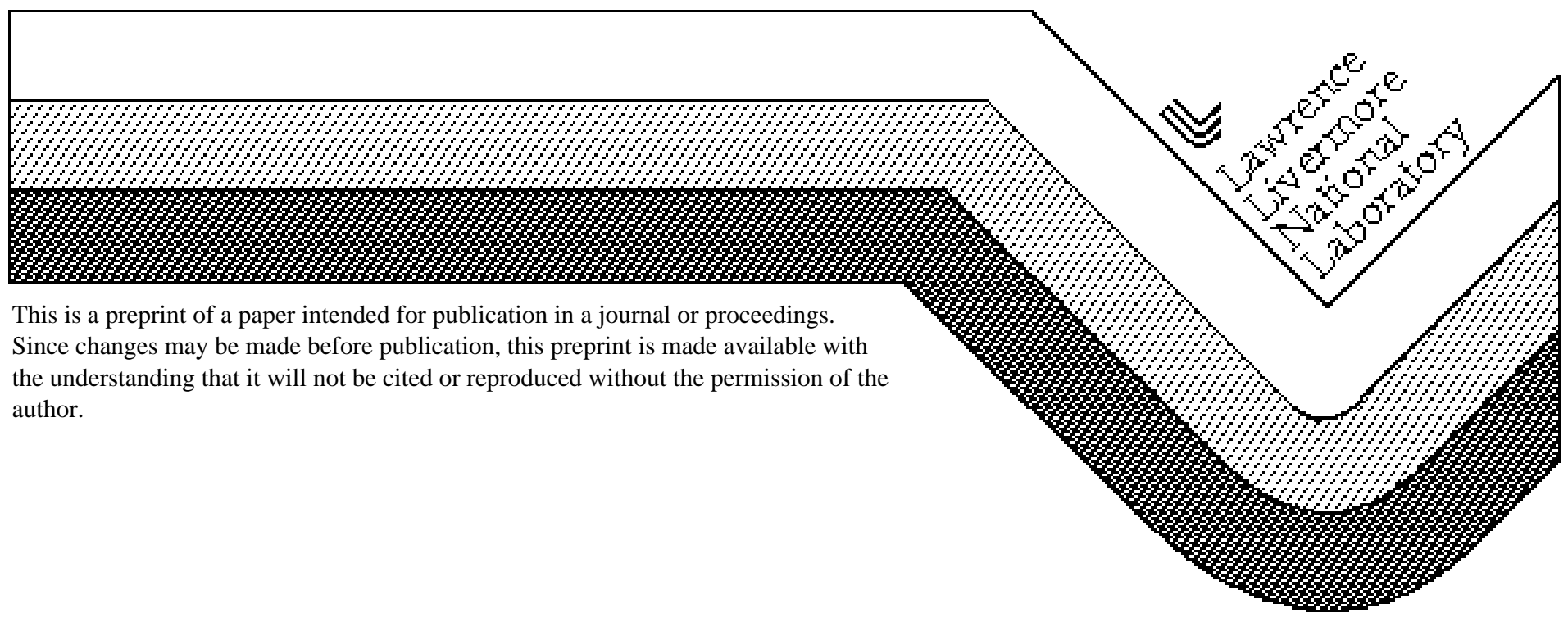




\section{DISCLAIMER}

This document was prepared as an account of work sponsored by an agency of the United States Government. Neither the United States Government nor the University of California nor any of their employees, makes any warranty, express or implied, or assumes any legal liability or responsibility for the accuracy, completeness, or usefulness of any information, apparatus, product, or process

disclosed, or represents that its use would not infringe privately owned rights. Reference herein to any specific commercial product, process, or service by trade name, trademark, manufacturer, or otherwise, does not necessarily constitute or imply its endorsement, recommendation, or favoring by the United States Government or the University of California. The views and opinions of authors expressed herein do not necessarily state or reflect those of the United States Government or the University of California, and shall not be used for advertising or product endorsement purposes. 
UCRL-JC-133744

\title{
Simulation of material removal efficiency with ultrashort laser pulses*
}

\author{
A.M. Komashko, M.D. Feit, A.M. Rubenchik, M. D. Perry, P.S. Banks \\ Lawrence Livermore National Laboratory \\ P.O. Box 808, mail stop L-477 \\ Livermore, CA 94550 USA \\ fax: $92-422-5537$
}

\section{Conference on Laser Ablation '99 \\ Goettingen, Germany}

July 19-23, 1999

email: komashko1@1lnl.gov, feit1@1lnl.gov, rubenchik1@1lnl.gov,banks1@1lnl.gov

\begin{abstract}
Understanding physical processes accompanying ablation is necessary for optimal use of ultrashort laser pulse (USLP) material processing. We describe the implementation of self-consistent electromagnetic propagation -energy absorption in our numerical models and estimate effect on material removal of energy, pulselength and prepulses.

\section{Introduction}

In the ultrashort pulse regime we are interested in, the initial hydrodynamic motion resulting from laser energy absorption is typically well described one dimensionally because the energy is deposited in a thin pancake shaped region. The width of the pancake is determined by the beamsize and the thickness is of the order of a skin depth (tens of nanometers). Physical insight into the ablation process can be enhanced through detailed numerical models[1,2] incorporating the relevant physics.
\end{abstract}


Thus, the simplest model of laser energy absorption is simply to declare that a certain fraction of the incident laser energy is deposited in a thin surface layer. This is sufficient for determination of some trends and regularities, but is, at best, only semiquantitative. Despite the one dimensional nature of the resulting hydrodynamics, the lasermaterial interaction depends strongly on the polarization of the laser light and its angle of incidence.

The next level of sophistication takes into account the index of refraction and absorption coefficient of the cold material to calculate Fresnel reflection and transmission coefficients. This gives explicit dependence on polarization and angle of incidence, but is still inadequate, in general, to treat quantitatively the laser energy absorption. The reason is that as laser energy is absorbed, plasma formation and expansion begin. Plasma ionization changes the electron density and expansion changes both the values of and the spatial distribution of refractive index which, in turn, modifies the absorption during later parts of the pulse. Even for ultrashort pulses of less than one ps duration, this effect can significantly affect the total pulse absorption as is shown below. Self consistency is even more important in understanding the effect of prepulses which create a pre-existant plasma profile for the main pulse.

Thus, a self consistent description involving both vector electromagnetic wave propagation and plasma hydrodyamics is necessary for reliable description of laser energy deposition. We have implemented such a description in the hydrocode HYADES. The incoming laser beam is propagated as a vector electromagnetic wave through a stratified (i.e. one dimensional) plasma profile.

Below, we describe, first, the numerical method used to propagate the electromagnetic field. We then present results from the integrated self consistent optical propagation hydrodynamic modeling for ultrashort laser pulse interaction with Al.

It is also intrinsically difficult to estimate theoretically the amount of material removal resulting from laser energy absorption. This is due to two reasons. 
Fundamentally, the initial density of deposited laser energy is extremely high. Response of the material involves hydrodynamic motion, shockwave generation and propagation, heat conduction, etc. Codes that describe these processes treat the material as a fluid. There is no model of material strength, fracture of cold material, etc. built into such models. Second, detailed simulations model a relatively short time during and following the lasermaterial interaction. Actual material removal occurs on longer time scales after the material cools down and phase transformations and fracture occur. For ultrashort pulses (less than $1 \mathrm{ps}$, say), we can carry our simulations out to hundreds of ns, but it is inadvisable to push such modeling too far. In addition to the fact that the model becomes less useful for cold materials at low pressures, three dimensional effects become important eventually. Thus, it is necessary to develop a short time theoretical criterion correlated with material removal. As described below, we use the "long time" zero velocity point to divide ablated from nonablated material. These simulations refer to the original material removal. At a later stage when holes of high aspect ratio are formed, considerations of waveguiding, wall absorption, material redeposition and surface instabilities must also be taken into account.

\section{Optical propagation model}

A medium with complex dielectric which varies only in the $\mathrm{z}$ direction can be treated as a stack of layers[3], each layer having a constant complex dielectric . A plane wave is incident on this medium with arbitrary angle of incidence and polarization. Satisfaction of the boundary conditions at each interface leads to a linear relationship between the fields in one layer to the next. That is, they are connected by a $2 \times 2$ matrix. The overal properties (transmission and reflection) of the medium are found by multiplying all these matrices together. The fields penetrating the medium and the resultant energy deposition (Joule heating) can then be simply found. The advantage to this approach is its arithmetical simplicity - it is ideally suited for numerical computation since it involves many simple operations. 


\subsection{Results of integrated laser absorption - hydrodynamic modeling}

The examples shown here refer mostly to aluminum since a reliable equation of state suited for the laser ablation regime is available to us.

If one holds the pulse duration fixed at $150 \mathrm{fs}$ and varies laser intensity, absorption begins to rise for fluences greater than about $1 \mathrm{~J} / \mathrm{cm}^{2}$ due to the high density "cold" plasma distribution. At very high fluences, however, the plasma becomes hot enough so that inverse Bremstrahlung absorption drops. This effect is shown in Fig.(1). It is instructive to look at the net absorption at low and moderate fluences as a function of angle of incidence (Fig.(2)). Absorption expected from the Fresnel coefficients calculated from the optical properties of cold $\mathrm{Al}$ are also plotted in the figure. It is seen that the low fluence pulse has absorption substantially the same as one expects from the cold material. However, at fluence of $16 \mathrm{~J} / \mathrm{cm}^{2}$, absorption is found to increase considerably at all angles of incidence and for both polarizations. We have carried out a similar numerical experiment for carbon. In this case, the cold absorption is high to begin with and the effect shown in Fig.(2) is not so dramatic.

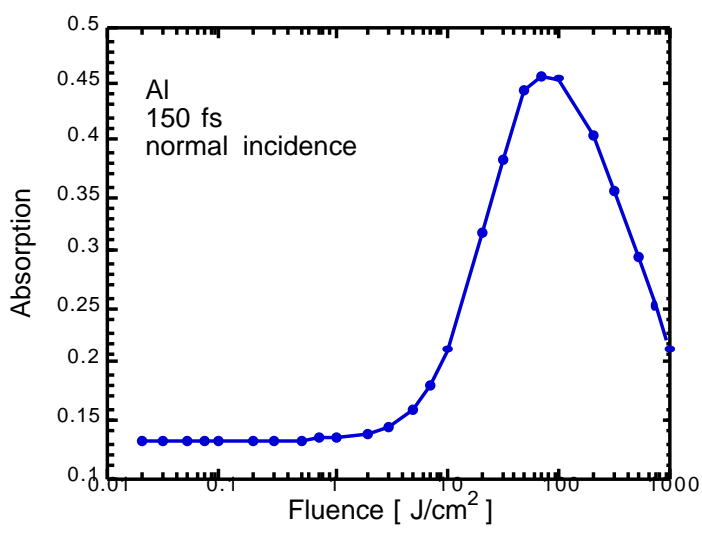

Fig(1) Fraction of normal incidence $150 \mathrm{fs}$ laser pulse absorbed by $\mathrm{Al}$ as function of energy fluence.

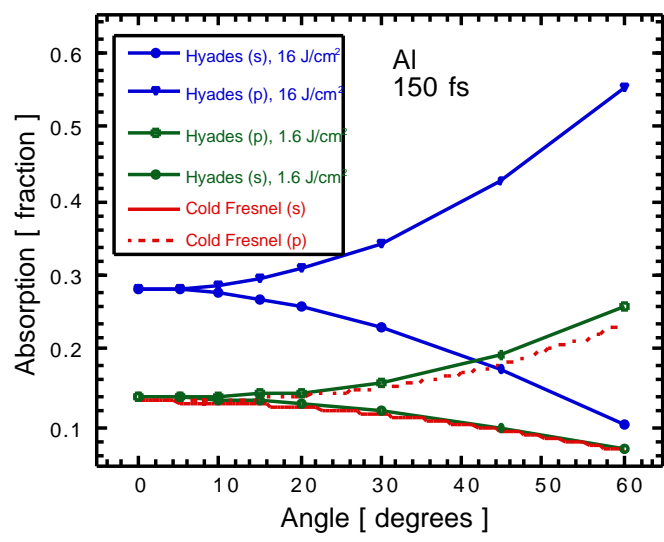

Fig(2): Net absorption of low fluence laser pulses can be estimated from cold Fresnel reflection and transmission coefficients. 
This is modified at high fluence through interaction of pulse with plasma.

\section{Material removal}

\subsection{Energy Efficiency}

We have used two short time estimators of ultimate material removal by laser ablation of a pristine surface. The first is the very natural approach of measuring the mass flow through a plane just outside the original material surface. While this yields a measure related to ultimate removal, the limited time over which the integration can be carried out results in absolute numbers that strongly underestimate actual values. We have found a better estimator to be given by locating the point at which zero material velocity occurs at times many pulselengths after the laser-material interaction. The idea is that all mass on one side of this point will leave the solid while that on the other represents the solid material motion leading to compression. In the following, we use this measure of mass removal via ablation. Note that this estimation neglects the effect of long time evaporation. This is most appropriate for metals because they cool rapidly.

We have used this criterion to carry out several systematic investigations of the energy efficiency of material removal with short laser pulses. Fig.(3) illustrates the transition from ultrashort to longer pulse ablation behavior. Here, the total energy absorption and material removed are calculated as a function of pulse width for a fixed energy fluence of $1.6 \mathrm{~J} / \mathrm{cm}^{2}$. Note that there is almost no dependence of either quantity on pulsewidth for pulses shorter than about 1 ps. For such short pulses, energy deposition is effectively instantaneous (thermal diffusion length and hydrodynamic expansion small compared to skin depth). For longer pulses, total absorption increases. However, most of this is in the expanding plasma and further removed from the solid surface for longer 
pulses. A much higher proportion of the absorbed energy ends up as waste heat for the longer pulses.

We have also examined theoretically the material removal rate as a function of fluence at fixed pulsewidth (Fig.(4)). Although the amount of material removed per pulse increases with incident fluence, the fluence specific removal rate,i.e. depth removed per unit incident fluence is calculated to be maximal just above the onset of appreciable material removal (see Fig.(4)). This point is not necessarily an optimal operating point for material processing, however, since it implies very many more pulses are necessary for a given amount of material removal than would be required at a higher, less energy efficient, incident fluence.

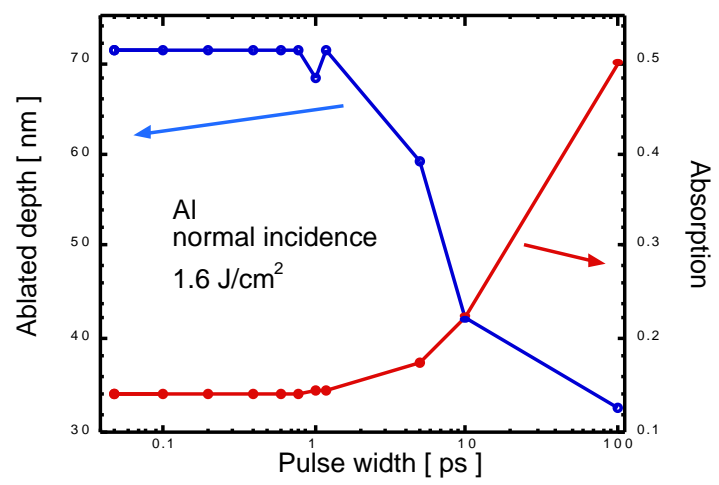

Fig.(3): Dependence of laser energy absorption and material removal on pulsewidth at constant fluence.

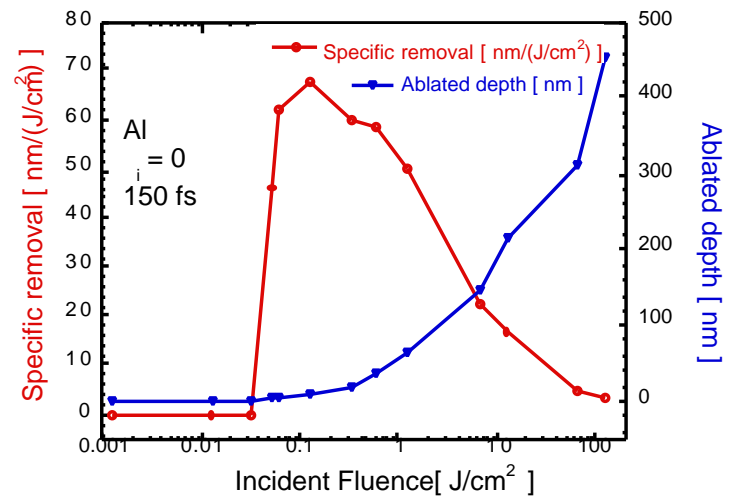

Fig.(4): Ablated depth and fluence specific initial material removal as a function of fluence for $150 \mathrm{fs}$ pulse on Al. The most energy efficient removal occurs at low fluences of about $0.1 \mathrm{~J} / \mathrm{cm}^{2}$.

\subsection{Prepulse}

From the absorption results given above, it is evident that, for $\mathrm{P}$ polarization, the usual inverse Bremstrahlung absorption can be supplemented and increased by resonance absorption provided a plasma of the right scale length (fraction of a wavelength) is available. Energy is now deposited away from the solid surface, but if absorption is increased enough, net material removal can also increase. This suggests material removal 
can be optimized by using a prepulse to create a plasma profile for the main pulse. An optimum exists with respect to timing between prepulse and main pulse since if the temporal separation is too long, laser energy is absorbed in the plasma far away from the solid and does not contribute to additional material removal. Our detailed calculations bear out this simple idea.

Laser energy absorption depends on both laser intensity and the lag between main and pre-pulses. Fig.(5a) shows the breakdown between inverse Bremstrahlung and resonance absorption for a $150 \mathrm{fs} 10^{13} \mathrm{~W} / \mathrm{cm}^{2}$ pulse with $10 \%$ prepulse. A broad peak in absorbed energy is noted. Fig.(5b) shows corresponding quantities for a pulse with 10 times the intensity and a 5\% prepulse. However, absorption does not correspond directly to material removal as noted above. The calculated material removal rate corresponding to conditions of Fig.(5a) is given in Fig.(6) where a definite optimum removal rate is seen.

Experimental results thus far do not show this enhancement of material removal rate. Our experimental studies of stainless steel showed a reduction of removal rate for all prepulse spacings. There are several possible reasons for this apparent difference. Experimental data refer to results of many pulses. Often the bottom of craters develop corrugations. With corrugations, the angle of incidence varies with position. In this case, there does not have to be an optimal prepulse timing and prepulses can result in a reduction in the amount of material removed. Our model calculations were done for $\mathrm{Al}$, a material with fairly low cold absorption. The preformed plasma considerably increases absorption and thus material removal. The experimental results are for stainless steel, a material with good cold absorption so the plasma enhancement effect is likely to be small as with carbon. In this case, the movement of the energy absorption site away from the surface will result in a decrease in material removal rate.

Unfortunately, our results do not reveal how to optimize practical large scale material removal, e.g. hole drilling. The reason is that once the hole begins to form, the hole walls are at different angles to the incident beam direction and polarization than was 
the original surface. Further, electric currents are generated in the walls which act as a laser energy absorber. This loss to the walls can be significant. Surface instabilities (corrugations) form which further modify the laser interaction with the solid material. Evaporation and recondensation on the walls may also be important.
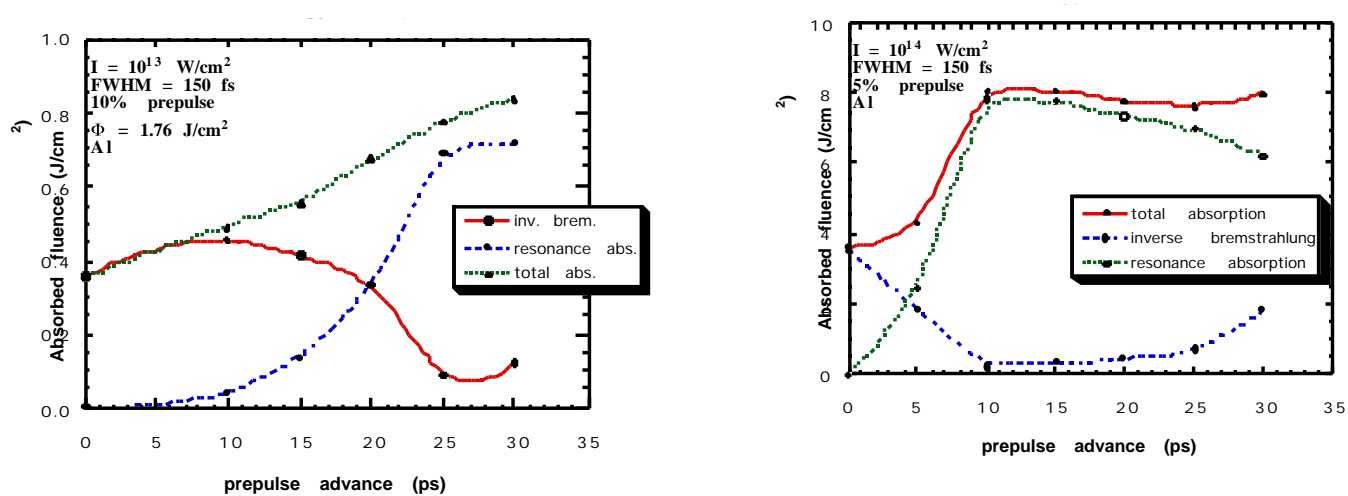

Fig.(5a): Decrease of inverse Bremstrahlung (5b): $10^{14} \mathrm{~W} / \mathrm{cm}^{2}, 5 \%$ prepulse. and increase of resonance absorption due to Note broad energy absorption peak. prepulse generated plasma profile. (a): $10^{13}$ $\mathrm{W} / \mathrm{cm}^{2}, 10 \%$ prepulse

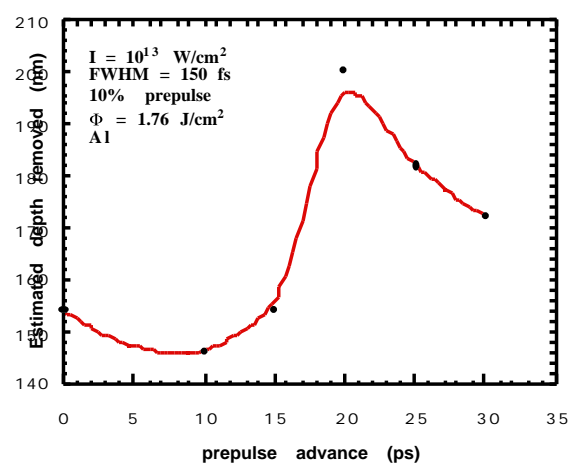

Fig(6): Material removal rate calculated for conditions of Fig.(6a) as function of 
temporal separation of main and prepulse.

At optimal prepulse timing, removal is

increased $33 \%$.

\section{Summary}

We formulated a vector optical propagation scheme accounting for laser beam polarization and angle of incidence. This scheme has been implemented into the one dimensional radiation-hydrodynamic code HYADES and permits a self consistent description of laser absorption and reflection from an expanding plasma. This is important for quantitative modeling since laser pulse absorption can be quite different from that expected from the "cold" Fresnel coefficients. We also developed theoretical estimates of ultimate material removal due to ablation. Detailed calculations demonstrated the high energy efficiency of material removal using ultrashort laser pulses and aids in determining optimal operational parameters.

* Work performed at Lawrence Livermore National Laboratory under the auspices of the U.S. Department of Energy under contract No. W-7405-ENG-48.

\section{references}

[1] "Physical Characterization of Ultrashort Laser Pulse Drilling of Biological Tissue", M.D. Feit, A.M. Rubenchik, B.-M. Kim, L.B. Da Silva, M.D. Perry, B.C. Stuart, Appl. Surf. Sci. 127-129, 869-74 (1998); "Numerical Simulation of ultra-short laser pulse energy deposition and transport for material processing", A. M. Rubenchik, M.D. Feit, M. D. Perry and J. T. Larsen, Appl. Surf. Sci. 127-129, 193-98 (1998) 
[2] "Ultrashort-Pulse Laser Machining of Dielectric Materials", M.D. Perry, B.C. Stuart,

P.S. Banks, M.D. Feit, V. Yanovsky, and A.M. Rubenchik, J. Appl. Phys. 85, 6803-10 (1 May 1999)

[3] M.Born, E.Wolf, Principles of Optics, Pergamon Press, (Oxford 1980) 